\title{
On the synergy of nuclear data for fusion and model assumptions
}

\author{
Vlad Avrigeanu ${ }^{\mathrm{a}}$ and Marilena Avrigeanu ${ }^{\mathrm{b}}$ \\ Horia Hulubei National Institute for Physics and Nuclear Engineering, PO Box MG-6, 077125 Bucharest-Magurele, Romania
}

\begin{abstract}
A deuteron breakup (BU) parametrization is involved within the BU analysis of recently measured reaction-in-flight (RIF) neutron time-of-flight spectrum, while open questions underlined previously on related fast-neutron induced reaction on $\mathrm{Zr}$ isotopes are also addressed in a consistent way, at once with the use of a recent optical potential for $\alpha$-particles to understand the large discrepancy between the measured and calculated cross sections of the ${ }^{94} \mathrm{Zr}(n, \alpha){ }^{91} \mathrm{Sr}$ reaction. Thus the synergy between the above-mentioned three distinct subjects may finally lead to smaller uncertainties of the nuclear data for fusion while the RIF neutron spectra may also be used to support nuclear model assumptions.
\end{abstract}

\section{Introduction}

The basic role of the improvement of nuclear data for fusion has recently been pointed out with respect to the overall uncertainties on the measured yield of reaction-inflight (RIF) neutrons with energies up to $30 \mathrm{MeV}$ produced in warm, dense deuterium-tritium plasma [1]. While the ultimate aim has been the improved cross sections for the reactions with thresholds above $15 \mathrm{MeV}$ in order to determine the RIF neutrons yield, a detailed analysis has been proved necessary for the quasimonoenergetic neutrons produced by the ${ }^{2} \mathrm{H}(d, n)^{3} \mathrm{He}$ reaction at deuteron energies from 15 to $19 \mathrm{MeV}$. Beyond the usual neutron energy spread, the deuteron breakup (BU) has been proved significant at these energies and a related fractional contribution (FC) were carefully determined from neutron time-of-flight (NTOF) spectra. Finally, on the basis of the ${ }^{90} \mathrm{Zr}(n, 2 n){ }^{89} \mathrm{Zr}$ monitor reaction cross-section evaluation with assumed uncertainties below $3.5 \%$ in the energy range studied, well-improved cross sections were obtained for certain reactions including ${ }^{94} \mathrm{Zr}(n, \alpha)^{91} \mathrm{Sr}$.

Several issues of this pioneering work [1] are considered hereafter, looking also for making use of their synergy within fusion physics, i.e. creation of a whole that is greater than the simple sum of its parts. First, a BU empirical parametrization [2] is involved within the BU analysis of the NTOF spectrum. Second, the open questions underlined by an widespread study of the fastneutron induced reaction on $\mathrm{Zr}$ isotopes [3], subsequent to the evaluation used formerly [1], are addressed in a consistent way (e.g., [4]). Third, a recent optical model potential (OMP) for $\alpha$-particles [5] is used to understand the large discrepancy between the measured $[1,3]$ and calculated [3] cross sections of the ${ }^{94} \mathrm{Zr}(n, \alpha){ }^{91} \mathrm{Sr}$ reaction. An improved physics modeling is thus leading to smaller uncertainties of the nuclear data for fusion while the RIF

\footnotetext{
a e-mail: vlad.avrigeanu@nipne.ro

b e-mail: marilena.avrigeanu@nipne.ro
}

neutron spectra may also be used to support nuclear model assumptions.

\section{Calculated BU neutron spectrum}

The quasimonoenergetic neutrons involved for the measurement of the ${ }^{169} \mathrm{Tm}(n, 3 n)^{167} \mathrm{Tm}$ reaction cross section from 17 to $22 \mathrm{MeV}$ [1] were produced by the ${ }^{2} \mathrm{H}(d, n)^{3} \mathrm{He}$ reaction at deuteron incident energies from 17.5 to $21.5 \mathrm{MeV}$. A careful attention was paid in particular to the deuteron breakup which contributes additional neutrons with lower energies from reactions in the target deuterium gas cell, entrance collimator, Havar entrance foil, and tantalum beam stop. Thus, in order to correct properly the activation by lower-energy neutrons, the neutron-energy distribution was determined at each energy setting by neutron time-of-flight (NTOF) measurements. Finally, the FC ratio of the activity induced from the breakup neutrons relative to the induced total activity, determined for each reaction of interest using excitation functions from nuclear data evaluations, was found to be more sensitive to uncertainties in the neutron-energy distribution than to differences between evaluated cross sections. The large FC values found even above 0.6 and 0.9 for the ${ }^{94} \mathrm{Zr}(n, \alpha){ }^{91} \mathrm{Sr}$ and ${ }^{169} \mathrm{Tm}(n, 2 n)^{168} \mathrm{Tm}$ reactions, respectively, may motivate further trials to establish the breakup neutron spectrum.

Thus, the BU cross sections may be obtained from an empirical parametrization [2] of both the total breakup nucleon-emission and elastic-breakup fractions, $f_{B U}^{n / p}=\sigma_{B U}^{n / p} / \sigma_{R}$ and $f_{E B}=\sigma_{E B} / \sigma_{R}$, respectively, where $\sigma_{R}$ is the deuteron total-reaction cross section. (The elastic breakup (EB) is the process in which the target nucleus remains in its ground state and none of the deuteron constituents interacts with it, while in the inelastic breakup or breakup fusion (BF) one of the deuteron constituents interacts nonelastically with the target nucleus.) Concerning the present case there is indeed a limitation given by the fact that this parametrization involved

(C) The Authors, published by EDP Sciences. This is an Open Access article distributed under the terms of the Creative Commons Attribution License 4.0 (http://creativecommons.org/licenses/by/4.0/). 


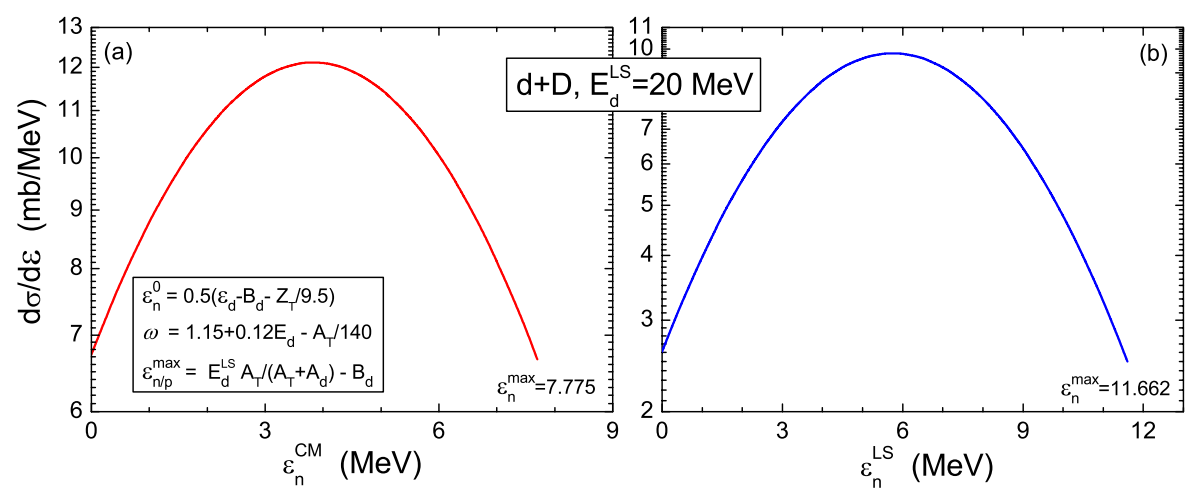

Figure 1. Calculated deuteron-breakup neutron spectrum in the (a) laboratory and (b) center-of-mass systems for deuterons incident on deuterium at $20 \mathrm{MeV}$, using the empirical parametrization [2] of both the total breakup nucleon-emission and elastic-breakup fractions to the deuteron total-reaction cross section for target nuclei from ${ }^{27} \mathrm{Al}$ to ${ }^{232} \mathrm{Th}$, and deuteron energies up to 80 and $30 \mathrm{MeV}$, respectively.

experimental $f_{B U}^{p}$ and $f_{E B}$ fractions for target nuclei from ${ }^{27} \mathrm{Al}$ to ${ }^{232} \mathrm{Th}$, for deuteron energies up to 80 and $30 \mathrm{MeV}$, respectively, while then it was applied and confirmed by consistent analysis of the deuteron induced reactions on target nuclei from ${ }^{27} \mathrm{Al}$ to ${ }^{223} \mathrm{~Pa}$ [6]. Therefore, the BU neutron spectrum shown in Fig. 1 and the corresponding fractions $f_{B U}^{n}=0.125$ and $f_{B U}=0.208$, for $20 \mathrm{MeV}$ deuterons incident on ${ }^{2} \mathrm{H}$, may provide only a first guess in this respect. Any further BU data for light target nuclei would be most helpful for a suitable extension of the above-mentioned BU parametrization [2].

\section{The fast-neutron induced reactions on $\mathrm{Zr}$ isotopes}

The above-mentioned work on the ${ }^{169} \mathrm{Tm}(n, 3 n)^{167} \mathrm{Tm}$ reaction cross sections just above $15 \mathrm{MeV}$ involved also two fast-neutron induced reactions on $\mathrm{Zr}$ isotopes, namely the ${ }^{90} \mathrm{Zr}(n, 2 n)^{89} \mathrm{Zr}$ monitor reaction used to determine the neutron flux by means of a recent evaluation [7], and the ${ }^{94} \mathrm{Zr}(n, \alpha){ }^{91} \mathrm{Sr}$ reaction. Actually, the particular importance in a wide range of applications and incident energies of the fast-neutron induced reactions on zirconium motivated also a relatively recent measurement and careful analysis of these reactions right above $15 \mathrm{MeV}$ [3]. There have been more question marks proved by that work on both the absolute cross sections and the excitation-function shapes, particularly for $(n, p)$ and $(n, \alpha)$ reactions. The related calculations were performed with version 1.0 of the computer code TALYS [8] using phenomenological models for direct interaction, pre-equilibrium emission (PE) and Hauser-Feshbach (HF) statistical equilibration of an excited nucleus, either by reusing as many as possible of the parameters optimized in the framework of assessing the available fast-neutron reaction data for ${ }^{89} \mathrm{Y}$, or globally optimized phenomenological model parameters as well as the important quantities that were derived from globally optimized microscopic calculations.

Alternatively, we pay attention firstly to the use of a local consistent input parameter set of (i) particle and (ii) $\gamma$-ray transmission coefficients, and (iii) back-shifted Fermi gas (BSFG) nuclear level densities [9]. They have been established or validated on the basis of independent measured data for neutron total cross sections and $(p, n)$ reaction cross sections [10], $\gamma$-ray strength functions
[11] and $(p, \gamma)$ reaction cross sections [10], and lowlying levels [12] and resonance data [13], respectively. The model calculations were carried out mostly using an updated version of the computer code STAPRE-H95 [14], with $\sim 0.4 \mathrm{MeV}$ equidistant binning for the excitation energy grid. The latest version 1.8 of the code TALYS [8] has also been used as well as the TALYS-based evaluated data library TENDL-2015 [15], for overall excitation function survey.

The setting up and validation of some of these local model parameters prove the need of additional reactions analysis. Thus, while the world-wide used optical potentials of Koning and Delaroche [16] were found suitable only for neutrons, even their local OMP parameter set for protons on ${ }^{90} \mathrm{Zr}$ overestimated significantly the ${ }^{89} \mathrm{Y}(p, n)^{89} \mathrm{Zr}$ reaction cross sections at the lowest energies. Actually the analysis of this reaction as well as of the ${ }^{89} \mathrm{Y}(p, \gamma)^{90} \mathrm{Zr}$ related channel in the same energy range, both of them being not fully described even within recent works [17], has proved to us most useful for the proper validation of the proton OMP of Johnson et al. [18] within a mass range well known for an anomalous OMP for sub-Coulomb protons [19].

The proper check of the proton transmission coefficients has provided confidence in the $(n, p)$ reaction cross sections calculated at the lower incident energies where only the discrete levels of the residual nuclei are populated. This additional confidence has really been needed due to the difference between various data sets beyond $1 \sigma$ error bars. The usefulness of these local setting up of model parameters by means of independent data is proved in Figs. 2 and 3 for the target nuclei ${ }^{90,94} \mathrm{Zr}$, by the agreement with different data sets of the present calculations and the evaluated data within the TENDL-2015 library which includes adjustments of the TALYS-1.8 results to various measured data [15], respectively.

While the previously detailed analysis of fast-neutron reactions on $\mathrm{Zr}$ isotopes [3] concluded correctly that the lack of agreement of the measured and their calculated cross sections especially for the $(n, p)$ reactions is due to PE effects, the present suitable description of both $(n, p)$ and $(n, 2 n)$ reactions on ${ }^{90} \mathrm{Zr}$ follows entirely the use of the PE Geometry-Dependent Hybrid (GDH) model [20] generalized through inclusion of the angular-momentum and parity conservation [21] and the $\alpha$-particle emission based on a pre-formation probability $\varphi[22]$ with the value 


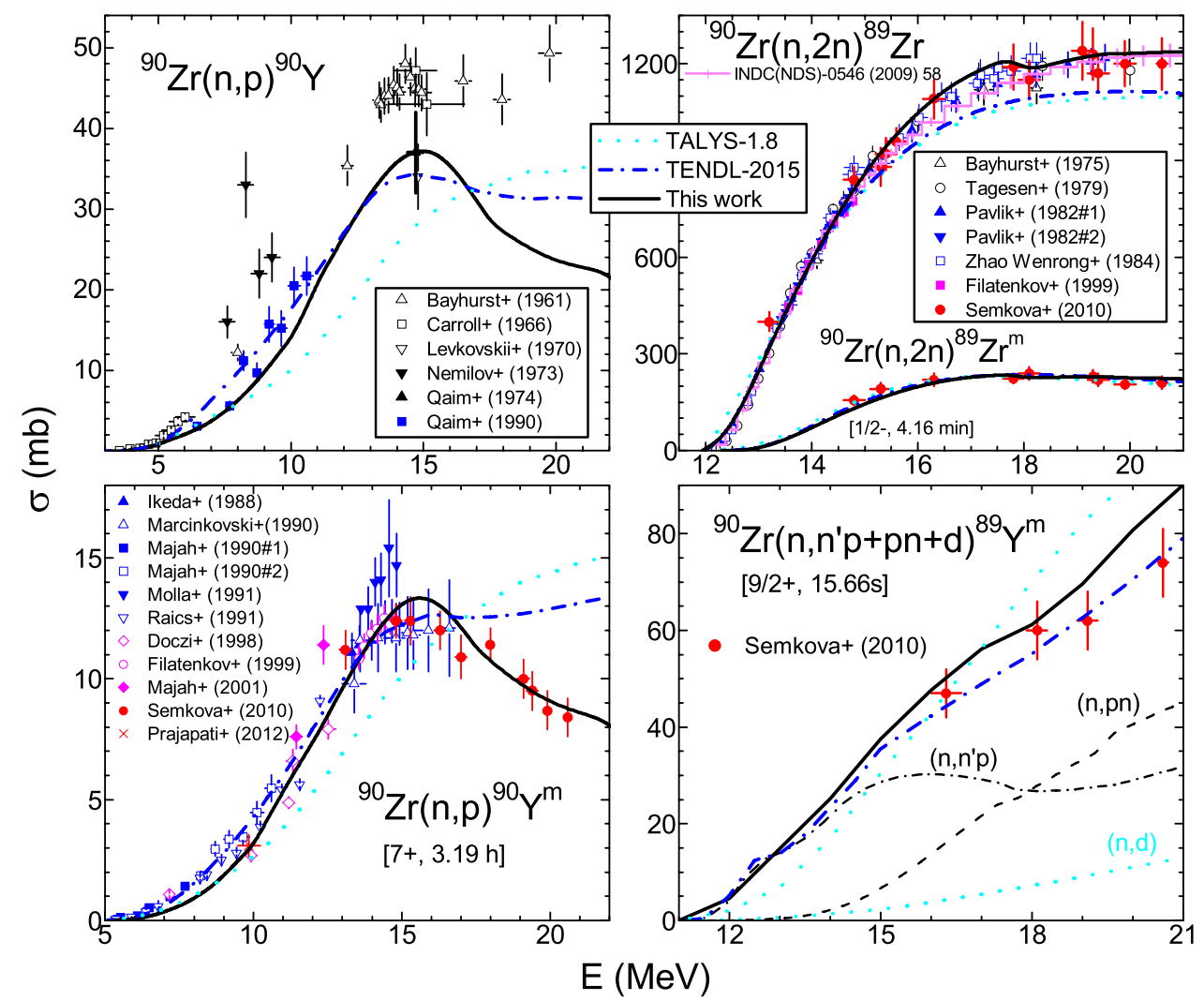

Figure 2. Comparison of the measured [3,10], global predictions of the code TALYS-1.8 [8] (dotted curves), evaluated [7,15] (dashdotted curves and histogram, respectively) and calculated (solid curves) fast-neutron reaction cross sections for ${ }^{90} \mathrm{Zr}$.

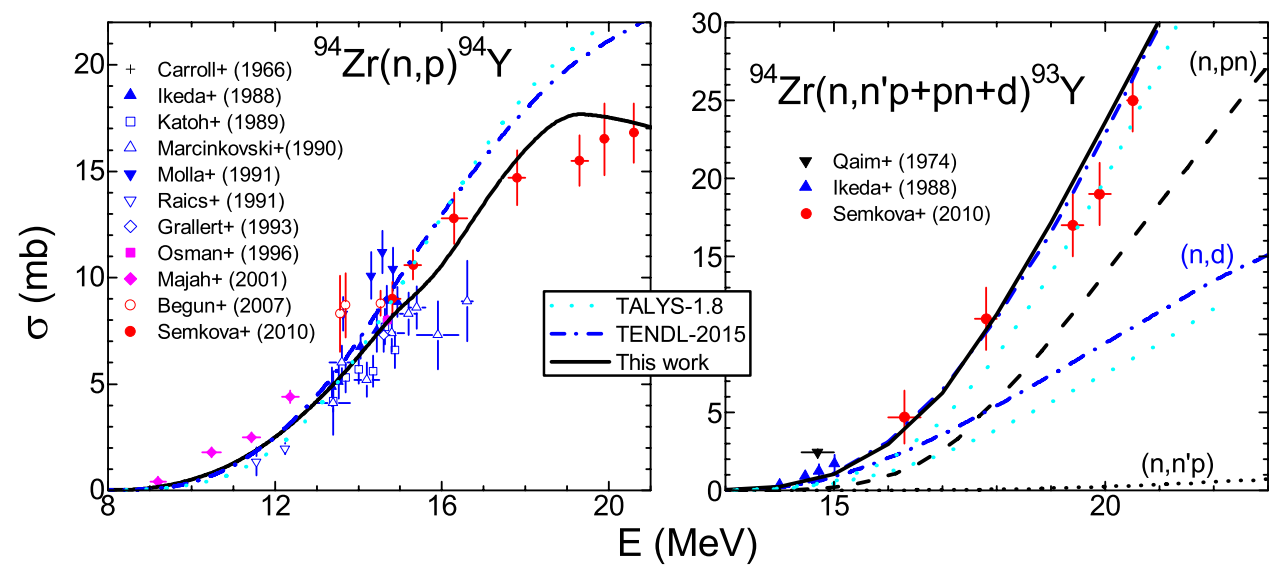

Figure 3. As Fig. 2 but for the target nucleus ${ }^{94} \mathrm{Zr}$.

0.2 in the present work. Thus, the increase of the neutron $\mathrm{PE}$ at the incident energy of $\sim 18 \mathrm{MeV}$ is due to the contribution of an additional partial wave (e.g., Fig. 4 of Ref. [23]). The similar effect for the proton PE happens at $\sim 20 \mathrm{MeV}$, a physical smoothing of the calculated cross sections in the energy range $18.1-21.5 \mathrm{MeV}$ leading to the shape of the $(n, p)$ excitation functions shown in Fig. 2.

\section{The $(n, \alpha)$ reaction account for $\mathrm{Zr}$ nuclei}

The analysis of the $(n, \alpha)$ reaction data available for the ${ }^{90,94} \mathrm{Zr}$ has proved a very different case, shown in Fig. 4. The use of the recent OMP for $\alpha$-particles [5], which provides a suitable description of the $\alpha$-particle induced reaction data within the wide mass range $45 \leq A \leq 209$, led to large discrepancy between the measured $[1,3,10]$ and calculated cross sections for both reactions.

Then, we replaced this potential by the one found to describe well the $\alpha$-particle evaporation [24] within two distinct steps. First, we did this replacement only for to obtain the $\alpha$-particle transmission coefficients involved in the HF calculations. The results have been in much better agreement with the measured data only at the lowest energies, where PE effects are not yet present. Second, we did the same replacement within the calculation of the corresponding PE intranuclear transition rates through the generalized GDH model, with the result of a rather good agreement. The remaining data underestimation around the incident energy of $12 \mathrm{MeV}$ could be due to a possible enhancement related to the position of a giant quadrupole resonance $(\mathrm{GQR})$ in these nuclei, similar to the case of 


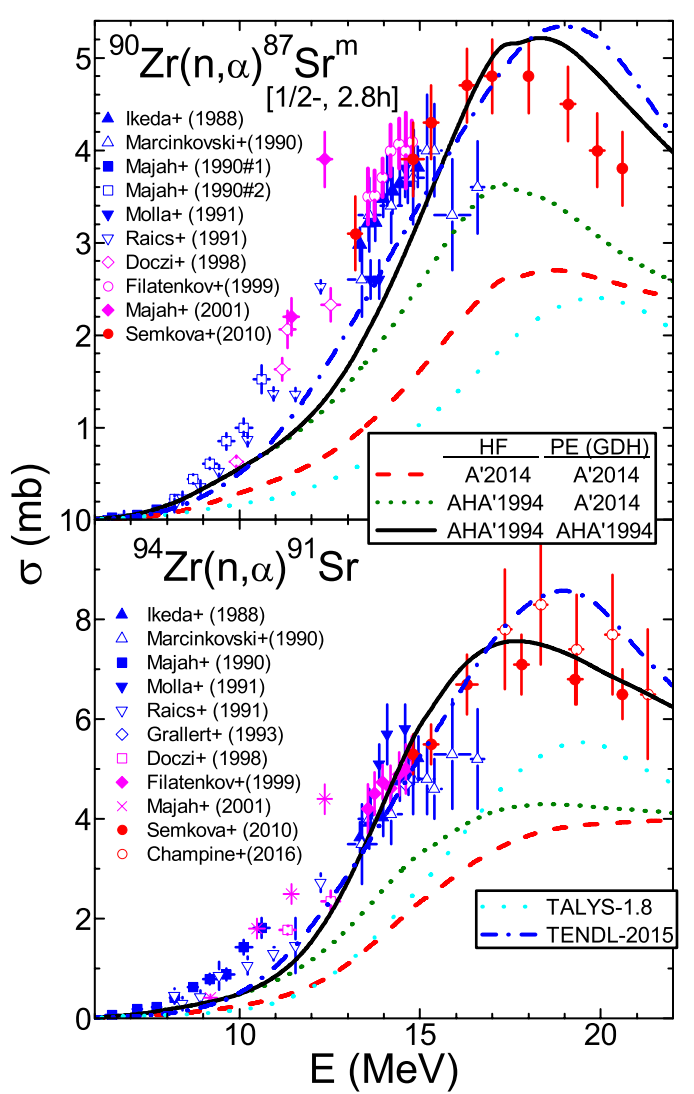

Figure 4. As Fig. 2 but for the $(n, \alpha)$ reaction on ${ }^{90,94} \mathrm{Zr}$, using the $\alpha$-particle global OMPs of Ref. [5] for either the HF and PE model calculations (dashed curves) or only the PE model (shortdotted curves), and that of Ref. [24] for either only the HF (shortdotted curves) or HF and PE model calculations (solid curves).

the Mo isotopes [25]. It results that the $\alpha$-particle OMP is however different in the incident and outgoing channels at least in the mass range $A \sim 90$ [25], while the opposite case has been found for $A \sim 60$ [26]. Therefore further similar work should concern other cases and mass ranges.

Finally it could be concluded that the synergy between the three distinct subjects - deuteron breakup, fast-neutron induced reactions, and $\alpha$-particle OMP - each of them making otherwise the object of challenging analysis, may provide answers to questions still open.

This work was partly supported by Fusion for Energy (F4E-GRT168-02) and Unitatea Executiva pentru Finantarea Invatamantului Superior, a Cercetarii, Dezvoltarii si Inovarii (Project No. PN-IIID-PCE-2011-3-0450).

\section{References}

[1] B. Champine et al., Phys. Rev. C 93, 014611 (2016)

[2] M. Avrigeanu et al., Fus. Eng. Design 84, 418 (2009)

[3] V. Semkova et al., Nucl. Phys. A 832, 149 (2010)

[4] M. Avrigeanu et al., Phys. Rev. C 85, 044618 (2012)

[5] V. Avrigeanu et al., Phys. Rev. C 90, 044612 (2014)

[6] P. Bém et al., Phys. Rev. C 79, 044610 (2009); E. Šimečková et al., ibid. 84, 014605 (2011); M. Avrigeanu et al., ibid. 85, 034603 (2012); ibid. 88, 014612 (2013); ibid. 89, 044613 (2014); ibid. 92, 021601(R) (2015); ibid. 94, 014606 (2016)

[7] K.I. Zolotarev, INDC(NDS)-0546 (IAEA, Vienna, 2009), p. 58

[8] A.J. Koning, S. Hilaire, and M.C. Duijvestijn, TALYS-1.8, Jan. 2016; http: //www . talys .eu

[9] H. Vonach et al., Phys. Rev. C 38, 2541 (1988)

[10] http://www-nds.iaea.org/exfor/

[11] A.C. Larsen et al., Phys. Rev. C 93, 045810 (2016); G.M. Tveten et al., ibid. 94, 025804 (2016)

[12] http://www.nndc.bnl.gov/ensdf

[13] R. Capote et al., Nucl. Data Sheets 110, 3107 (2009); http://www-nds. iaea.org/RIPL-3/

[14] M. Avrigeanu and V. Avrigeanu, IPNE Report NP86-1995, Bucharest, 1995, and Refs. therein; News NEA Data Bank 17, 22 (1995)

[15] A.J. Koning and D. Rochman, Nucl. Data Sheets 113, 2841 (2012); A.J. Koning et al., https:// tendl.web.psi.ch/tendl_2015/tendl2015.html

[16] A.J. Koning and J.P. Delaroche, Nucl. Phys. A713, $231(2003)$

[17] L. Netterdon et al., Nucl. Instr. Meth. in Nucl. Res. A 754, 94 (2014); S. Harissopulos et al., Phys. Rev. C 87, 025806 (2013)

[18] C.H. Johnson, R.L. Kernell, and S. Ramavataram, Nucl. Phys. A 107, 21 (1968)

[19] C.H. Johnson, A. Galonsky, and R.L. Kernell, Phys. Rev. C 20, 2052 (1979)

[20] M. Blann and H.K. Vonach, Phys. Rev. C 28, 1475 (1983); M. Blann, Nucl. Phys. A 213, 570 (1973)

[21] M. Avrigeanu, M. Ivascu, V. Avrigeanu, Z. Phys. A 329, 177 (1988); ibid. 335, 299 (1990)

[22] E. Gadioli, E. Gadioli-Erba, Z. Phys. A 299, 1 (1981)

[23] P. Reimer et al., Phys. Rev. C 71, 044617 (2005)

[24] V. Avrigeanu, P.E. Hodgson, and M. Avrigeanu, Phys. Rev. C 49, 2136 (1994)

[25] M. Avrigeanu, W. von Oertzen, and V. Avrigeanu, Nucl. Phys. A 764, 246 (2006)

[26] V. Avrigeanu and M. Avrigeanu, Phys. Rev. C 91, 064611 (2015) 